\title{
Task-Specific Dynamics For Robotic Hand Control
}

\author{
Eric Rombokas, Mark Malhotra, Yoky Matsuoka
}

\begin{abstract}
Humans employ context-specific internal models for their bodies and the world around them. They learn them through embodiment in the environment, by gathering data in a task-specific way. Similarly, robots operating under humanlike task constraints must have the ability to accommodate situations unknown to their designers. We demonstrate a method for the Anatomically Correct Testbed (ACT) robotic hand to use task-relevant data to build a reduced-dimensionality controller tailored to that task. The robotic hand encounters a novel task and models the combined dynamics of robot and environment. This is achieved without additional model complexity, and without prior knowledge of the task. We show the utility of this approach for playing a piano key, attaining a single-key trill at the maximum speed allowed by the piano dynamics. This task is chosen because it is relatively simple from a kinematic perspective, mostly involving flexion-extension, but is quite complex from a dynamic perspective, including a contact transition.
\end{abstract}

\section{INTRODUCTION}

Robotic hand research has led to a variety of approaches to enable manipulation capabilities, but has been limited by the complexity of system and task demands. The best-performing robotic hands are still limited to specific hardwired tasks and require controlled environments.

Neuroscience researchers have uncovered aspects of how humans are able to perform multiple tasks, learn new tasks through experience, and make use of the rich complexities of the hand. This knowledge can be used to control robotic hands by reducing the dimensionality of the problem and learning task-specific dynamics through experience.

\section{A. IMPROVING ROBOTIC HAND CONTROL}

Researchers have enabled robotic hands to perform tasks previously beyond the reach of industrial process robots, such as high-speed object catching [1]. While this works remarkably well for this specific task, it is hard to generalize this technique to enable multiple complex tasks whose details may be unknown a priori.

In order to perform a variety of tasks without changing hardware, robotic manipulators can draw inspiration from human hands, in both their shape and control strategies. Researchers have attained solutions to biologically plausible hand simulations [2] [3], modeling the biomechanical properties of tendons, muscles [4] and fingers [5].

In choosing which features to model, we must consider the "Curse Of Dimensionality," [6] and the tradeoff between modeling task-significant phenomena and combinatorially complex computational demands. The human brain appears to use context-specific control strategies, modeling only the features that are pertinent to that context, so this could be a promising approach for robotic hand control as well. Studies

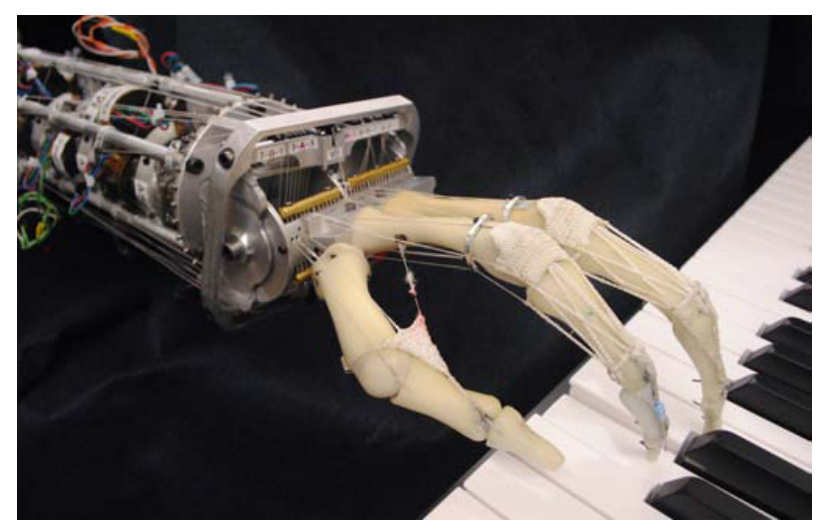

Fig. 1. Anatomically Correct Testbed (ACT) hand

of motor behavior indicate that humans don't adapt a single one-size-fits-all internal model of the body and environment, but instead build a repertoire of movement strategies that depend on context [7] [8] [9].

Previous work has achieved reduction of model complexity by expert construction [10] or by fitting the full model and intelligently pruning it down [11]. Researchers have approached robotic walking by manually splitting the task into two phases, with corresponding task-relevant properties considered in controllers for each [12], or by manually splitting the task into simultaneous subsystems [13]. We employ synergies, a concept developed in the study of biological movement control, to reduce the dimensionality of the system representation, and then we learn a dynamic model for that simplified representation.

\section{B. SYNERGIES}

The hand is controlled by over 30 muscles and has at least 22 degrees of freedom. Complex tendon relationships create a kinematic and dynamic situation with redundancy and nonlinearities that can become difficult to model. Modeling all the details is a problem with many dimensions and the system is always changing due to fatigue and growth. Despite this, infants start using their hands in a dynamic way very early in their lives. This has led researchers to search for simplifying principles that could help to explain this extraordinary capability.

Muscle synergies are generally a tool of analysis. Researchers record data from a session, sometimes general behavior but more typically repetitions of a task, using EMG, neural recordings, kinematic measurements, etc. This data is decomposed into a representation that spans the manifold of choices made in the larger space of possibilities. Basis 
functions are generated by methods such as PCA and nonnegative matrix factorization [14], whether static or explicitly including time [15] or the use of tools [16].

What simplifying assumptions make sense, given imperfect knowledge of future task constraints and environmental dynamics? A simple representation of the full musculoskeletal system should reflect the regularities in the context of the desired posture and task [17].

\section{OUR APPROACH}

We used a controller based on a synergy system approximation to control only the task of playing the piano, instead of the general movement problem. Embodiment of the robotic system in the environment provided the information necessary for synergy construction and control.

First we created synergies defined in tendon space to gather data to make an initial estimate of task dynamics. These synergies were derived from demonstration for this experiment, but could be generated by any data-gathering scheme capabable of roughly exploring the task kinematics. This technique does not require a complete system specification or measurement of high-level features like joint angles.

We learned a dynamics model in terms of the contextspecific synergies, and compare the results to a model-free feedback controller, and a model built using general (rather than task-specific) motion.

\section{SYSTEM AND METHODS}

\section{A. ACT HAND}

The value of a context-specific controller will be most apparent when controlling a robot which exhibits contextspecific kinematics and dynamics. The ACT Hand (Figure 1) mimics the interactions among muscle excursions and joint movements produced by the bone geometries and tendon connectivity of the human hand. This mimicry results in a high-dimensional system with the redundancies and nonlinearities of the biological hand [18] [19].

The ACT hand uses 24 motor-driven tendons to control a thumb, index finger, middle finger, and wrist. Each segment of these fingers is machined using human bone data, and is accurate in surface shape, mass, and center-of-gravity to the human equivalent. The extensor mechanisms are complex webs of tendons on the dorsal side of the fingers, and are crucial for emulating dynamic human behavior [20]. As each tendon is pulled by a motor, it is routed through attachment points mimicking human tendon sheaths and following the contours of the bones. Since these bone shapes are complicated surfaces, the effective moment arm the tendon exerts on the joint varies with joint angle [21].

In a tendon-driven system like the human hand, each tendon must pull and not push, and therefore require $n+1$ tendons to fully actuate $n$ degrees of joint freedom. Even with this requirement, the joints of the human (and ACT hand) are redundant. For instance, there are six tendons controlling the four degrees of freedom of the index finger. Three of these tendons are relatively simple: one is an extensor and two are flexors, attached to different segments but having only a

\begin{tabular}{l|ccccr} 
& I & B & K & F1 & F2 \\
\hline Finger & 0.5445 & 114.52 & 510.46 & 402.2 & -195.8 \\
Task & 0.5763 & 168.46 & 6284.4 & 1348.7 & -315.09
\end{tabular}

TABLE I

Model PARAMETERS: INERTIA (I), DAMPING (B), STIFFNESS (K), AND FRICTION IN THE POSITIVE (F1) AND NEGATIVE (F2) DIRECTIONS.

slightly nonlinear dependence on the state of the finger. The situation for the remaining three tendons, however, is highly nonlinear and state-dependent. They affect each joint very differently depending on the posture of the finger, not just in the magnitude of their moment arms on the joints, but even in the direction.

\section{B. CONSTRUCTION OF SYNERGY AND MODEL}

Self-experience data was collected by demonstration. A human moved the index finger of the ACT hand, causing it to tap a piano key 30 times, and the resultant tendon lengths were recorded at $200 \mathrm{~Hz}$. Synergies for this experiment took the form of linear weightings of motor activations. Given the demonstration data, Principal Component Analysis (PCA) revealed the dimensions which best account for the observed variability in tendon lengths $l$. Using only the first component as our task synergy, $67.3 \%$ of the variance of the session was preserved, which is sufficient to perform the dynamic task of tapping the piano key quickly. Note that the synergy was constructed using only the excursions of the tendons, and required no modeling of dynamic properties or force measurements.

The synergy provided a vector of weights, $s \in \mathbb{R}^{n}$, for each of the $n$ motor/encoder combinations that can be used for reduced dimensionality state estimation and control. The synergy angle, denoted $\theta_{s}$, can now be considered a virtual joint angle defined by: $\theta_{s}=s^{T} l$. Similarly, the synergy torque $\tau_{s}$ acts as a virtual joint torque, such that desired torques on the virtual joint can be achieved by calculating $u=\tau_{s} s$, where $u$ are the motor torques.

Whereas a muscle excursion controller would require $n$ simultaneously running PID controllers, the dimensionality reduction achieved by the synergy $s$ allows a single PID controller to track $\theta_{s}$ using $\tau_{s}$ for actuation. However, this method is subject to the limitations of PID control which especially degrade performance at high speeds. Incorporating a feedforward term that anticipates known dynamics can alleviate this problem and improve high-speed performance.

We assumed a model of the form:

$$
\tau_{s}=I \ddot{\theta}_{s}+B \dot{\theta}_{s}+K \theta_{s}+F 1\left(\dot{\theta}_{s}>0\right)+F 2\left(\dot{\theta}_{s}<0\right)
$$

where $I, B, K, F 1$, and $F 2$ are parameters expressing the contributions of inertia, damping, stiffness, positive friction, and negative friction respectively. Contrary to models that consider each muscle's effect on each joint's motion, this synergistic model composes together muscles and joints according to their interactions.

The model parameters were identified for the key-tapping synergy in two cases: 1) finger-only : the index finger was 


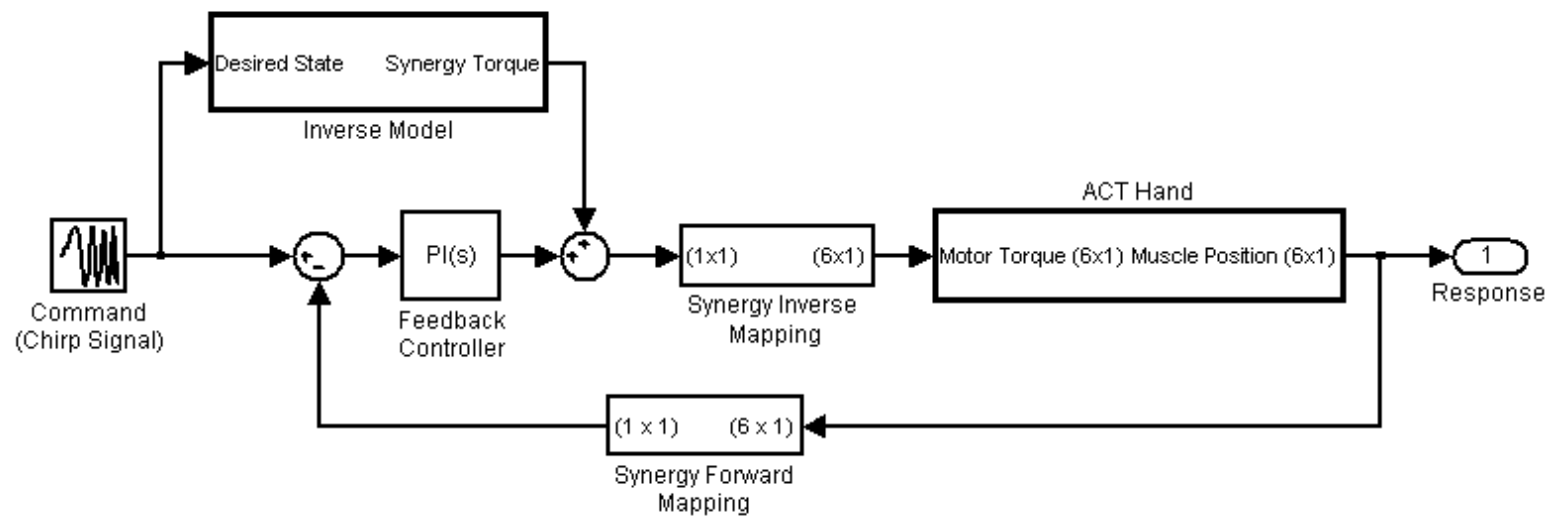

Fig. 2. Feedforward Controller Architecture

in air, experiencing only its own dynamics and 2) task : the index finger was moving along with the piano key, experiencing the combined dynamics of the finger and the piano key. During each case, the finger is commanded to a desired trajectory of a sin wave with linearly increasing frequency. The identified parameters are shown in Table I. As expected, combining the dynamics of the finger and piano together increases all parameter values, indicating that the piano has inertia, damping, stiffness, and friction. The presence of the piano key contributes significantly to the system dynamics, but is not explicitly modeled with additional model structure. Instead, the finger and piano key are considered a single task-specific system.

\section{CONTROLLERS}

A proportional-integral (PI) feedback controller was tuned for maximum performance while still guaranteeing stability. Due to delay, noise, and actuator limitations, a feedback controller will typically exhibit poor performance at high speeds. Adding a feedforward term allows the controller to work proactively, anticipating known dynamics. The controller diagram in Figure 2 depicts the architecture of the synergistic feedforward control.

\section{EXPERIMENT}

The experiments evaluate the three controllers: 1) feedback only, 2) feedforward using the finger-only dynamic model, and 3) feedforward using the task dynamic model. During each experiment, the finger is placed on the piano key and the controller is commanded to follow the desired synergy angle $\theta_{s, D}$ : a sine wave of frequency increasing from 0.1 $\mathrm{Hz}$ to $6.81 \mathrm{~Hz}$ within 30 seconds, and with the amplitude of a full keystrike. This trajectory provides uniform excitation for the frequencies of interest, up to the maximum speed of key release in order to differentiate the frequency responses of each controller.

During the experiment, tendon excursions are recorded at $200 \mathrm{~Hz}$ and converted into observed synergy angles, $\theta_{s, O}$. The synergy angle tracking error is defined as $\varepsilon_{\theta}=$ $\theta_{s, D}-\theta_{s, O}$. Each experiment is separated into ten evenly-
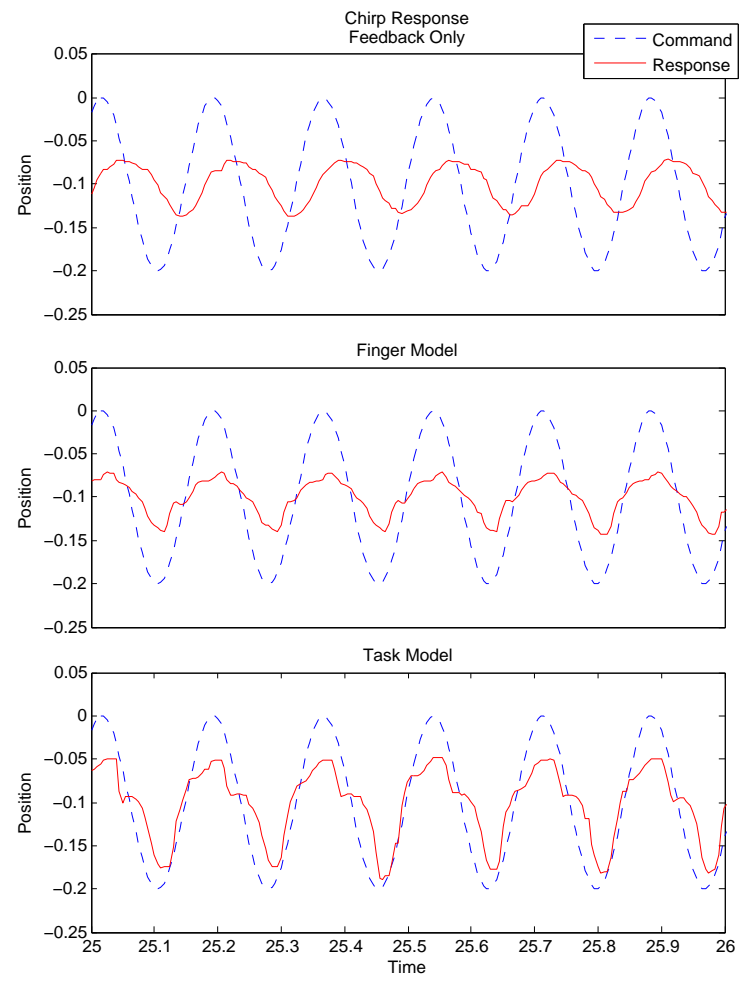

Fig. 3. A section of each controller's response for the fast portion of the trill $(5 \mathrm{~Hz})$. The traces depict the synergy angles during a representative trial for the feedback-only, finger-model, and task-specific controllers, from top to bottom.

spaced frequency bands and the root mean squared error is calculated as a measure of frequency dependent performance.

\section{RESULTS}

As expected, the model-based controllers outperform the simple feedback controller, and the task model outperforms the finger-only model. Two-factor ANOVA was used to assess the effect of controller type and frequencies ( 10 frequency bands, linearly spaced from $0.1-6.81 \mathrm{~Hz}$, see Figure 4 ), followed by all pairwise comparisons of controllers in each frequency band, using 30 two-sample two- 


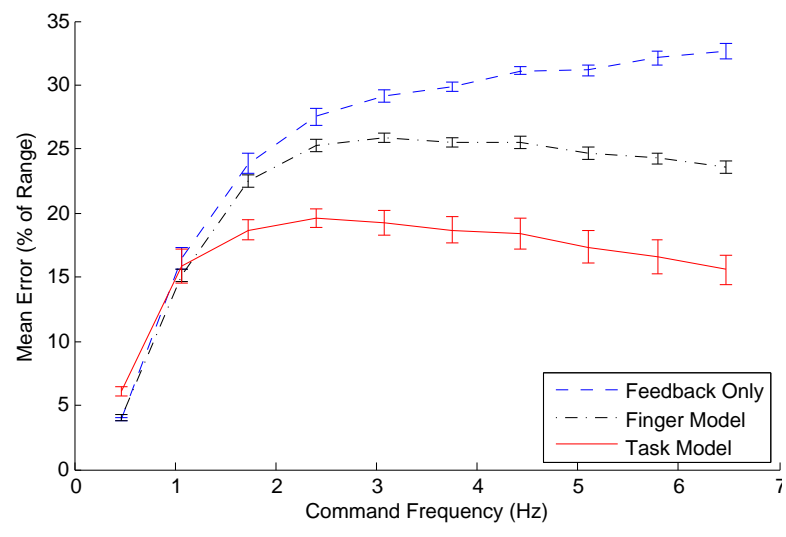

Fig. 4. Error for each controller. Feedforward performance improves for faster movement, consistent with the dynamics model capturing properties such as damping.

tailed Student's t-test. The effects of alpha inflation were mitigated using the Bonferroni Correction, choosing a family error rate of 0.05 .

The two-factor ANOVA showed a statistically significant effect of controller on error $(\mathrm{p}<0.001)$, and frequency $(\mathrm{p}$ $<0.001)$. For pairwise differences in controller performance for bands 3-10 $(1.39-6.81 \mathrm{~Hz})$, all tests were significant, with the task-specific model outperforming the finger-only model outperforming the feedback-only controller. Results for frequencies slower than this were mixed, indicating that the benefit of using models is only apparent for faster operating speeds.

Figure 3 illustrates the responses of the three controllers near the maximum speeds. The increasing benefit of using the finger-only and task contollers can be seen in Figure 4.

\section{DISCUSSION}

The improved performance of the task-specific controller did not require additional model complexity, and was built from a novel experience, the details of which could not have been known beforehand. Like the human motor control system, this allows the robot to model a novel task given limited experiential knowledge. Such a method enables both the flexibility and task precision required for robots interacting with a unknown or changing environment. Further development of this method is required to accommodate multiple simultaneous task constraints or complex kinematic situations, such as multiple fingers working in concert.

Here we demonstrate an improvement of robotic control using neuroscience inspiration, but continued work may shed light in the reverse direction. Since the ACT hand is biologically comparable to the human hand, comparison of the learning performed using particular methods may inform the quest to understand human motor learning.

\section{ACKNOWLEDGEMENTS}

We thank Cara Stepp for experimental design discussion and comments on the manuscript.

\section{REFERENCES}

[1] A. Namiki, Y. Imai, M. Ishikawa, and M. Kaneko, "Development of a high-speed multifingered hand system and its application to catching," in Intelligent Robots and Systems, 2003.(IROS 2003). Proceedings. 2003 IEEE/RSJ International Conference on, vol. 3, pp. 2666-2671, IEEE, 2004.

[2] E. Theodorou, E. Todorov, and F. Valero-Cuevas, "Neuromuscular Stochastic Optimal Control of a Tendon Driven Index Finger Model,"

[3] A. Bicchi and D. Prattichizzo, "Analysis and optimization of tendinous actuation for biomorphically designed robotic systems," Robotica, vol. 18 , no. 1 , pp. $23-31,2000$.

[4] I. Brown and G. Loeb, "Measured and modeled properties of mammalian skeletal muscle: IV. Dynamics of activation and deactivation," Journal of muscle research and cell motility, vol. 21, no. 1, pp. 33-47, 2000.

[5] N. Pollard and R. Gilbert, "Tendon arrangement and muscle force requirements for human-like force capabilities in a robotic finger," in ICRA'02, vol. 4, pp. 3755-3762, IEEE.

[6] N. Bernstein, The co-ordination and regulation of movements, vol. 1. Pergamon Press Oxford:, 1967.

[7] M. Haruno, D. Wolpert, and M. Kawato, "Multiple paired forwardinverse models for human motor learning and control," in Advances in neural information processing systems 11: proceedings of the 1998 conference, p. 31, The MIT Press, 1999.

[8] N. Malfait and D. Ostry, "Is interlimb transfer of force-field adaptation a cognitive response to the sudden introduction of load?," Journal of Neuroscience, vol. 24, no. 37, p. 8084, 2004.

[9] D. M. Wolpert, R. C. Miall, and M. Kawato, "Internal models in the cerebellum," Trends in Cognitive Sciences, vol. 2, no. 9, pp. $338-$ 347, 1998.

[10] I. Walker, D. Dawson, T. Flash, F. Grasso, R. Hanlon, B. Hochner, W. Kier, C. Pagano, C. Rahn, and Q. Zhang, "Continuum robot arms inspired by cephalopods," in Proc. 2005 SPIE Conf. Unmanned Ground Vehicle Technology IV, pp. 303-314, Citeseer.

[11] M. Berniker, A. Jarc, E. Bizzi, and M. Tresch, "Simplified and effective motor control based on muscle synergies to exploit musculoskeletal dynamics," Proceedings of the National Academy of Sciences, vol. 106, no. 18, p. 7601, 2009

[12] M. Stilman, C. Atkeson, J. Kuffner, and G. Zeglin, "Dynamic programming in reduced dimensional spaces: Dynamic planning for robust biped locomotion," in Robotics and Automation, 2005. ICRA 2005. Proceedings of the 2005 IEEE International Conference on, pp. 23992404, IEEE, 2006.

[13] E. Whitman and C. Atkeson, "Control of a walking biped using a combination of simple policies," in Humanoid Robots, 2009. Humanoids 2009. 9th IEEE-RAS International Conference on, pp. 520-527, IEEE.

[14] A. d'Avella, P. Saltiel, and E. Bizzi, "Combinations of muscle synergies in the construction of a natural motor behavior," Nature Neuroscience, vol. 6, no. 3, pp. 300-308, 2003.

[15] A. d'Avella and M. Tresch, "Modularity in the motor system: decomposition of muscle patterns as combinations of time-varying synergies," in NIPS, pp. 141-148, 2001.

[16] M. Santello, M. Flanders, and J. Soechting, "Postural hand synergies for tool use," Journal of Neuroscience, vol. 18, no. 23, p. 10105, 1998

[17] E. Todorov and Z. Ghahramani, "Unsupervised learning of sensorymotor primitives," in Engineering in Medicine and Biology Society, 2003. Proceedings of the 25th Annual International Conference of the IEEE, vol. 2, pp. 1750-1753, IEEE, 2004.

[18] V. Weghe, M. Rogers, M. Weissert, and Y. Matsuoka, "The ACT hand: design of the skeletal structure," in Robotics and Automation, 2004. Proceedings. ICRA'04. 2004 IEEE International Conference on, vol. 4, pp. 3375-3379, IEEE, 2004.

[19] A. Deshpande, J. Ko, D. Fox, and Y. Matsuoka, "Anatomically correct testbed hand control: muscle and joint control strategies," in Robotics and Automation, 2009. ICRA'09. IEEE International Conference on, pp. 4416-4422, IEEE, 2009.

[20] D. Wilkinson, M. Weghe, and Y. Matsuoka, "An extensor mechanism for an anatomical robotic hand," in Robotics and Automation, 2003. Proceedings. ICRA'03. IEEE International Conference on, vol. 1, pp. 238-243, IEEE, 2003.

[21] A. Deshpande, R. Balasubramanian, R. Lin, B. Dellon, and Y. Matsuoka, "Understanding variable moment arms for the index finger MCP joints through the ACT hand," in Biomedical Robotics and Biomechatronics, 2008. BioRob 2008. 2nd IEEE RAS \& EMBS International Conference on, pp. 776-782, IEEE, 2009. 